\title{
Interference and Outage in Mobile Random Networks: Expectation, Distribution, and Correlation
}

\author{
Zhenhua Gong and Martin Haenggi
}

\begin{abstract}
In mobile networks, distance variations caused by node mobility generate fluctuations in the channel gains. Such fluctuations can be treated as another type of fading besides multi-path effects. In this paper, the interference statistics in mobile random networks are characterized by mapping the distance variations of mobile nodes to the channel gain fluctuations. The network performance is evaluated in terms of the outage probability. Compared to a static network, the interference distribution in a single snapshot does not change under uniform mobility models, but random waypoint mobility increases the interference. Furthermore, due to the correlation of the node locations, the interference and outage are temporally and spatially correlated. We quantify the temporal correlation of the interference and outage in mobile Poisson networks in terms of the correlation coefficient and conditional outage probability, respectively. The results show that it is essential that routing, MAC, and retransmission schemes be smart (i.e.. correlationaware) to avoid bursts of transmission failures.
\end{abstract}

Index Terms-Correlation, interference, mobility, Poisson point process.

\section{INTRODUCTION}

\subsection{Motivation}

In wireless networks, interference is one of the central elements in system design, since network performance is often limited by competition of users for common resources [1]. There are four major sources of randomness that affect the interference in large networks. The first is multi-path fading, which is the time variation of the channel strengths due to smallscale effects. The second one is node placement. In mobile networks, a random model of spatial locations is necessary to facilitate the network analysis. A wellaccepted model for the node distribution in wireless networks is the homogeneous Poisson point process (PPP) [2], [3], where the number of nodes in a certain area of size $A$ is Poisson distributed with parameter $\lambda A$, where $\lambda$ is defined as node intensity. The numbers of nodes in disjoint areas are mutually independent. The third one is power control, which helps in the interference management, energy optimization, and

- The authors are with the Wireless Institute, Department of Electrical Engineering, University of Notre Dame, Notre Dame, IN 46556, USA. connectivity [4], [5]. When power control is implemented locally, the receiver is not aware of the power levels of other interfering transmitters and transmit levels become a source of randomness in wireless networks. In this paper, however, we do not consider power control. The fourth one is data traffic. ALOHA [6] and CSMA [7] are two classes of well-accepted random and distributed medium access control (MAC) protocols.

For the sake of mathematical tractability and simplicity, the statistics of the above four sources of randomness are often assumed identically and independently distributed (i.i.d.). For example, the channels are often assumed to be memoryless; if mobility is at all considered, the nodes are highly mobile so that the realizations of node locations are independent in different time slots; the node activities are not affected by previous activities. Are those assumptions realistic? In wireless networks, the i.i.d. assumptions for multi-path channel realizations, transmit power levels, and data traffic statistics are reasonable, if nodes transmit in short bursts. Furthermore, some broadband transmission techniques, such as frequency-hopping spread-spectrum, nullify the channel memories as well. For node placement, however, the situation is different. The correlation between node locations in different time slots is zero only if a completely new realization of the node placement is drawn in each time slot. Network models assuming independent realizations are impractical since the node velocities cannot be infinite. If the node placement follows a certain type of distribution such as a PPP in each time slot and the nodes do not have infinite mobility, the node locations in different time slots are correlated. An extreme case is a static but random network, where the correlation is equal to one, since nodes do not move after their initial placement.

How does mobility affect network structure and performance? First, it is well known that multi-path fading is induced by microscopic mobility. A slight position change of a node induces randomness in channel gain. On the other hand, when distance is considered in wireless transmission, a significant change in the transmission distance, macroscopic mobility, 
gives rise to another degree of uncertainty: pathloss uncertainty. In this paper, we denote the multipath fading simply as fading, and large-scale path-loss uncertainty as large-scale fading. Both types of fading are induced by mobility. Second, mobility induces temporal and spatial correlation. Unless the node speed is infinite, the locations of a node always show a certain degree of correlation in different time slots. The quantification of such correlation is important, since it greatly affects the network performance.

\subsection{Related work}

There is a growing body of literature of large wireless networks with randomly distributed nodes. Stochastic geometry [8] and the theory of random geometric graphs [9] are two increasingly widely used analysis tools, which have been summarized in [2]. Interference and outage statistics are obtained in the case where nodes are Poisson distributed without multi-path fading [10], [11] and in the presence of fading [12], [13]. For the node placement models other than homogeneous Poisson, distance statistics in finite uniformly random networks are obtained in [14]. Interference and outage in clustered ad hoc networks are discussed in [15]. Interference results for ad hoc networks with general motioninvariant node distribution are presented in [16]-[18]. The interference distribution in doubly Poisson cognitive networks is analyzed in [19]. In [20], the hardcore point process is approximated by a non-homogeneous PPP to evaluate the outage. The performance of spatial relay networks is analyzed in [21], [22]. Routing in ad hoc networks is discussed in [12], [23]-[25]. The throughput and capacity in interference-limited networks have been derived in [26]-[28]. The spatio-temporal correlation of the interference and outage in static random networks has been studied in [29]. The spatial distribution of link outages in static random networks has been derived in [30].

Related work on mobile networks includes [31], where a network of mobile nodes is mapped to a network of stationary nodes with dynamic links. In [32], different mobility models and their effects to ad hoc networks are compared. Stochastic properties of random walk and random waypoint mobility models are analyzed in [33] and [34]-[37], respectively. Another way of combining micro- and macroscopic path loss uncertainty has been explored in [38], where small-scale fading is interpreted as a distortion of the point process in modeling the node locations.

\subsection{Our contribution}

The main contributions of this paper are:

1) We characterize interference and outage statistics in mobile random networks and investigate the affects of different mobility models such as constrained i.i.d. mobility (CIM), random

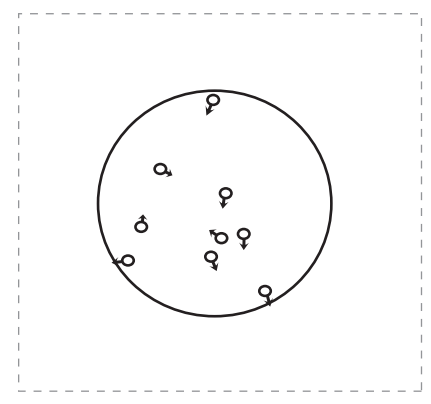

(a) Finite network

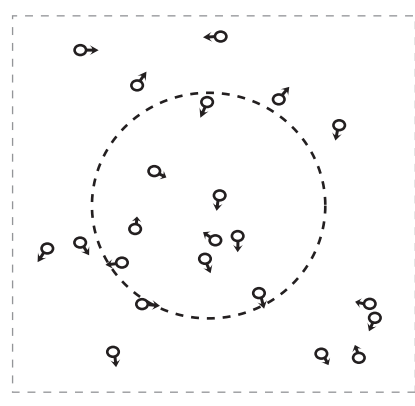

(b) Infinite network
Fig. 1: Illustrations of finite and infinite mobile networks. The small circles denote mobile nodes, and the arrows show the directions in which they will move in the next time slot. In (a), the nodes bounce back when they reach the boundary. In (b), all nodes move freely.

walk (RW), Brownian motion (BM), and random waypoint (RWP) to the network performance.

2) We quantify the temporal correlation of interference and outage in mobile random networks, with concrete results on correlation coefficient of interference and conditional outage probability, respectively.

The rest of the paper is organized as follows. System and mobility models are introduced in Section 2. In Section 3, the single-snapshot analysis of the interference and outage in mobile random networks is discussed. The temporal correlation of the interference and outage is analyzed in Section 4. Conclusions are presented in Section 5 .

\section{SYSTEM MODELS}

\subsection{Network model}

We consider the link between a typical transmitterreceiver pair in a wireless network. Without loss of generality, the link distance is normalized to one. We set the origin $o$ at the receiver. The initial node placement follows a Poisson point process $\Phi(0)$ on a domain $\mathbb{D}$ with intensity $\lambda$. The Poisson model implies the independence of the numbers of the nodes in disjoint regions. In a finite network as shown in Fig. 1 (left), $\mathbb{D}=B(o, R)$, where $B(o, R)$ is a disk of radius $R$. The number of nodes $M$ inside $B(o, R)$ is Poisson distributed with mean $\lambda \pi R^{2}$. In an infinite network as shown in Fig. 1 (right), $\mathbb{D}=\mathbb{R}^{2}$.

At any time, all nodes move independently of each other by updating their positions at the beginning of each time slot. In a finite network, the nodes bounce back when they reach the boundary so that $M$ remains constant. In an infinite network, all nodes move freely. Hence, the locations of potential interferers follow a homogeneous or non-homogeneous PPP $\Phi(t)=\left\{x_{i}(t)\right\}$ at any time $t \in \mathbb{N}_{0}$.

\subsection{Mobility models}

Different mobility models lead to different spatial properties of the networks and, in turn, affect the 
network performance differently [32]. In this part, we introduce several well-accepted models in the literature. For a fair comparison between different models, we first define the average speed of the nodes and set it to the same level. The speed of node $i$ in one time slot is defined as $v_{i}(t)=\left\|x_{i}(t)-x_{i}(t-1)\right\|$, where $t \in \mathbb{N}_{0}$ and $\|\cdot\|$ is the Euclidean distance. We define

$$
\bar{v} \triangleq \mathbb{E}\left[v_{i}(t)\right] \text {. }
$$

$\bar{v}$ is the mean speed averaged over all nodes, or equivalently, over all times for a fixed node. Here the time slot is measured at the time scale of mobility, which is indicated Fig. 2(a). $\bar{v}$ is much larger than the radio signal wavelength. The communication time scale, which will be introduced in the next subsection, is at the level of mobility or much shorter. Due to ergodicity and node homogeneity, the space averages are equal to the time averages.

\subsubsection{Constrained i.i.d. mobility (CIM)}

The constrained i.i.d. mobility (CIM) model is first introduced in [31]. Here, we consider an identical model except for the first time slot at $t=0$. The node location $x_{i}(t)$ is

$$
x_{i}(t)=y_{i}+\bar{v} w_{i}(t)
$$

where the home locations of the nodes $\Psi=\left\{y_{i}\right\}$ form a PPP, and $w_{i}(t)$ is uniformly at random in $B\left(y_{i}, \bar{v} R_{\mathrm{CIM}}\right)$. Using results from [37], we calculate the normalized mobile range $^{1} R_{\mathrm{CIM}}=45 \pi / 128 \approx 1.1045$. The CIM model is non-Markov. However given $y_{i}, x_{i}(t)$ and $x_{i}(t+s)$ are i.i.d. for all $s>0$.

\subsubsection{Random walk (RW)}

Under the random walk (RW) model, a mobile node selects a new direction and speed randomly and independently in each time slot. Hence, the spatial node distribution remains uniform [33]. Mathematically, the location of node $i$ at time $t+1$ for $t \in \mathbb{N}_{0}$ is

$$
x_{i}(t+1)=x_{i}(t)+\bar{v} w_{i}(t),
$$

where the distribution of $w_{i}(t)$ is uniformly at random in $B\left(x_{i}(t), \bar{v} R_{\mathrm{RW}}\right)$. The normalized mobile range $R_{\mathrm{RW}}=$ 1.5 , which is straightforward. In finite networks, the node is bounced (reflected) back when it meets the boundary ${ }^{2}$.

\subsubsection{Discrete-time Brownian motion (BM)}

Under the discrete-time BM model, the node location at time $t+1$ for $t \in \mathbb{N}_{0}$ is

$$
x_{i}(t+1)=\left(x_{i, 1}(t+1), x_{i, 2}(t+1)\right),
$$

where $x_{i, 1}(t+1)=x_{i, 1}(t)+\bar{v} w_{i, 1}(t)$ and $x_{i, 2}(t+$ $1)=x_{i, 2}(t)+\bar{v} w_{i, 2}(t)$, where $w_{i, 1}(t)$ and $w_{i, 2}(t)$ are

1. The term "normalized" means that the average node speed is equal to one.

2. We consider the same border behavior as presented in [34].

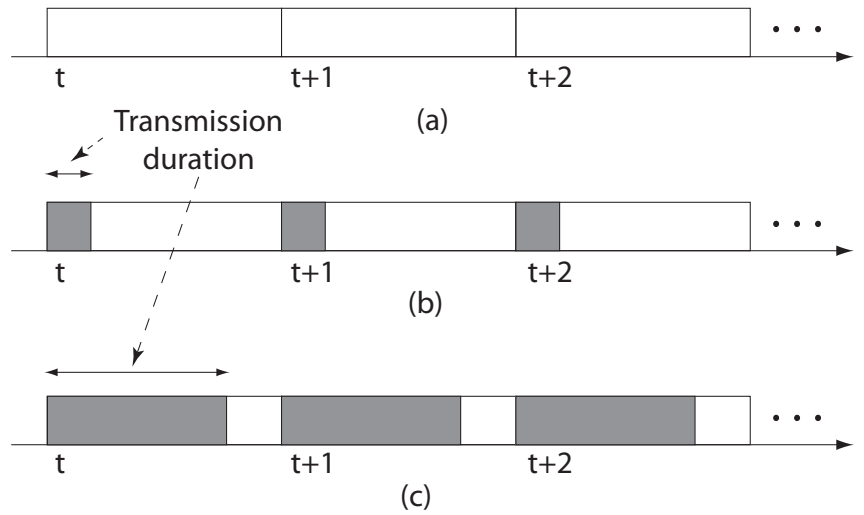

Fig. 2: Mobility and transmission time scales. The mobility (time) slots are indicated in (a). If a node is assigned for transmission, each transmission period, which is presented in gray, is assumed to start at the beginning of each mobility slot. In (b), the transmission duration is much shorter than the mobility (time) slot; in (c), the length of transmission time is comparable to the mobility slot.

i.i.d. normally distributed i.e., $w_{i, 1}(t), w_{i, 2}(t) \sim \mathcal{N}\left(0, \sigma^{2}\right)$. After normalization, we have $\sigma=\sqrt{2 / \pi}$.

Remark. From [12], [33, Lemma 2.2], and [39], the above mobility models preserve the uniform properties of the node distributions. Consequently for any $t$, the PPP $\Phi(t)$ remains homogeneous, if $\Phi(0)$ is homogeneous.

\subsubsection{Random waypoint (RWP)}

This model is only strictly defined in a finite area. Nodes are uniformly placed at $t=0$. Then, each node uniformly chooses a destination in the area and moves towards it with randomly selected speed $^{3}$. A new direction and speed are chosen only after the node reaches the destination. Otherwise, it keeps the same direction and speed for several time slots. After a long running time, its spatial node distribution converges to a non-uniform steady distribution [35].

\subsection{Channel access scheme}

We assume that the transmission starts at the beginning of each time slot, and each transmission is finished within one time slot. Slotted ALOHA is assumed as the MAC protocol. In every time slot $t$, each node determines whether to transmit or not independently with probability $p$. The transmission ends before the end of the mobility (time) slot as shown in Fig. 2(b) and 2(c). The next transmission (if the node is assigned to transmit) starts at the beginning of successive mobility (time) slot. This channel access scheme minimizes the magnitude of the correlation. Note that this model is also suitable for the case where not all transmissions start at

3. In the simulations, the speed is chosen so that the total distance is a multiple of the speed. 
the beginning of a mobility time slot; since spreading out the transmissions using time division scheduling reduces the density of interferers, this case is modeled by reducing the transmit probability $p$ by the appropriate factor.

\subsection{Channel model}

The attenuation in the wireless channel is modeled as the product of a large-scale path gain component and a small-scale fading gain component. The large-scale path loss function $g(x)$ is given by

$$
g(x)=\frac{1}{\epsilon+\|x\|^{\alpha}}, \quad \epsilon \geqslant 0,
$$

where $\alpha$ is the path loss exponent. Two categories of models are usually considered: the singular path-loss model where $\epsilon=0$ and the non-singular path-loss model where $\epsilon>0 . g(x)$ is assumed to be square integrable, i.e.,

$$
\int_{\nu}^{\infty} g^{2}(x) \mathrm{d} x<\infty, \quad \forall \nu>0 .
$$

In a two-dimensional network, $\alpha>2$ is necessary and sufficient to satisfy the integrability condition.

For the multi-path fading, we consider a deterministic model (i.e., no fading) and the Rayleigh and Nakagami fading models in the desired link and the interfering links. In Rayleigh fading, the pdf of the power fading gain $h$ is given by

$$
f_{h}(x)=\exp (-x)
$$

In the more general Nakagami- $m$ fading model, the $\mathrm{pdf}$ of the power fading gain is given by

$$
f_{h}(x)=\frac{m^{m} x^{m-1} \exp (-m x)}{\Gamma(m)} .
$$

If the transmission duration is relatively long, i.e., comparable to the length of the time slot (Fig. 2(c)), the packet may observe a large number of realizations, since the node covers many wavelengths in distance. With interleaving, the fading will then have a negligible effect only. On the other hand, if the transmissions are short (Fig. 2(b)), fading needs to be accounted for using the Rayleigh or Nakagami models. We consider both cases in this paper.

\subsection{Total interference}

Generally, the power received at the receiver from an interferer is given by

$$
P_{\mathrm{R}}=P_{\mathrm{T}} h_{x} g(x),
$$

where $P_{\mathrm{T}}$ is the transmit power, assumed normalized to 1 and $h_{x}$ is multi-path fading gain. At time $t$, the total interference at the receiver is

$$
I(t)=\sum_{x \in \Phi(t)} T_{x}(t) h_{x}(t) g(x)
$$

where $T_{x}(t)$ is i.i.d. Bernoulli with parameter $p$ due to ALOHA, and $h_{x}(t)$ has mean $\mathbb{E} h=1$.

\subsection{Outage probability}

The outage probability $p_{o}$ is one of the fundamental performance metrics in wireless networks. In interference-limited channels, an outage occurs if the signal-to-interference ratio (SIR) at a receiver is lower than a certain threshold $\theta$ i.e., $p_{o} \triangleq \mathbb{P}(\operatorname{SIR}<\theta)$.

\section{SINGLE-SNAPSHOT ANALYSIS OF INTER- FERENCE AND OUTAGE}

In this section, we evaluate the network performance in a single snapshot of time. We assume that $\epsilon=0$. The non-singular model $\epsilon>0$ can be treated similarly. The mobility models in Section 2.2 are separated into two categories: uniform and non-uniform.

\subsection{Interference in uniformly mobile networks}

Because of the uniformity of the mobility, the network in any time $t$ can be treated as correlated realizations of a static network. Hence the existing results of the interference and outage statistics in static networks in [5], [10] also apply to uniformly mobile networks.

\subsection{Interference in non-uniformly mobile networks}

\subsubsection{Interference in finite networks without fading}

We consider RWP and set $\mathbb{D}=B(o, R)$. Since we are only interested in the interference distribution in a single time slot, we can drop the dependence on $t$ and focus on the generic random variable

$$
I=\sum_{x \in \Phi} T_{x}\|x\|^{-\alpha}
$$

There are no closed-form pdf expressions of the interference in most cases. However, since the received power decays according to a power law, only considering the interference from the nearest interferer to the receiver provides a good approximation if the path-loss exponent $\alpha$ is not too close to 2 [5]. Therefore, we have the interference power approximately as

$$
I \approx I_{1}=R_{1}^{-\alpha},
$$

where $R_{1}$ is the distance between the origin to its nearest interferer. For RWP mobility, the node distance distribution in a circular region is [35]

$$
f_{L}(r)=\frac{1}{R^{2}}\left(-\frac{4 r^{3}}{R^{2}}+4 r\right) .
$$

Given a realization of the total number of nodes $M$, we have

$$
\begin{aligned}
\mathbb{P}\left(R_{1} \leqslant r \mid M\right) & =1-\left(1-F_{L}(r)\right)^{M} \\
& =1-\left(1-\left(\frac{2 r^{2}}{R^{2}}-\frac{r^{4}}{R^{4}}\right)\right)^{M} .
\end{aligned}
$$


Since $M$ is Poisson distributed with mean $\lambda \pi R^{2}$, the pdf of $R_{1}$ is thus given by

$$
\begin{aligned}
f_{R_{1}}(r) & =\frac{\mathrm{d} \mathbb{E}_{M}\left[\mathbb{P}\left(R_{1} \leqslant r \mid M\right)\right]}{\mathrm{d} r} \\
& =p \lambda \pi\left(4 r-4 \frac{r^{3}}{R^{2}}\right) e^{-p \lambda \pi\left(2 r^{2}-\frac{r^{4}}{R^{2}}\right)} .
\end{aligned}
$$

From (9) and (12), we obtain the pdf of $I_{1}$ :

$$
f_{I_{1}}(x)=2 p \lambda \pi \delta\left(x^{-\delta-1}-\frac{x^{-2 \delta-1}}{R^{2}}\right) e^{-p \lambda \pi\left(2 x^{-\delta}-\frac{x^{-2 \delta}}{R^{2}}\right)} .
$$

With deterministic channels, a simple lower bound on the outage probability is derived using the nearestinterferer approximation:

$$
\begin{aligned}
p_{o}^{\mathrm{nf}}(\theta) \triangleq \mathbb{P}\left(\frac{1}{I}<\theta\right) & \geqslant \mathbb{P}\left(\frac{1}{I_{1}}<\theta\right) \\
& =1-F_{I_{1}}\left(\theta^{-1}\right) \triangleq \underline{p}^{\mathrm{nf}}(\theta) .
\end{aligned}
$$

Calculating explicitly,

$$
\underline{p}^{\mathrm{nf}}(\theta)=1-\exp \left(-p \lambda \pi\left(2 \theta^{\delta}-\frac{\theta^{2 \delta}}{R^{2}}\right)\right) .
$$

\subsubsection{Interference in finite networks with multi-path fading}

When the interferers' channels are subject to multi-path fading, the interference power from the nearest interferer is $h_{1} I_{1}$, where $h_{1}$ is the multi-path fading coefficient. Then

$$
\underline{p}^{\mathrm{f}}(\theta)=\mathbb{E}_{I_{1}}\left[\mathbb{P}\left(\frac{h}{h_{1} I_{1}}<\theta \mid I_{1}\right)\right],
$$

where $h$ is the fading gain in the desired link. In the Rayleigh fading case, the cdf of $H \triangleq h / h_{1}$ is

$$
F_{H}(x)=\frac{x}{x+1} .
$$

A lower bound of outage probability is thus given by

$$
\underline{p}^{\mathrm{f}}(\theta)=\int_{0}^{\infty} \frac{x f_{I_{1}}(x)}{(x \theta+1)^{2}} \mathrm{~d} x .
$$

The lower bounds of the outage probabilities and the simulation results are plotted in Fig. 3. For comparison, the lower bounds and simulation results under the RW model are also included. The expected number of nodes in the region $\mathbb{E} M=10 \pi \approx 31$. From the figure, we find that the nearest-interferer approximation provides a close approximation in terms of the outage probability, in particular in the lower threshold regime, which is the regime of practical interest. Furthermore, multi-path fading is harmful to the link connections in mobile networks.

\subsubsection{Interference in infinite networks}

In infinite networks, the RWP model cannot be properly defined. However, we can derive the Laplace transform of the total interference if the node distance distribution follows (10). The Laplace transform of the interference is first calculated under a finite radius $R$, and then we let $R \rightarrow \infty$. Since the mobility model itself can not be defined, such a result is not the interference characterization under the RWP model in infinite networks, but it provides an asymptotic expression as $R$ gets large.

Proposition 1. For $R \rightarrow \infty$, the Laplace transform of the total interference converges to

$$
\mathcal{L}_{I}(s)=\exp \left(-2 \pi \lambda p s^{\delta} \mathbb{E}\left[h^{\delta}\right] \Gamma(1-\delta)\right) .
$$

Proof: We start with finite networks with radius $R$. From (10), the intensity measure of the point process is

$$
\Lambda(B(o, r)) \triangleq \mathbb{E}(\Phi(B(o, r)))=2 \lambda \pi r^{2}-\frac{\lambda \pi r^{4}}{R^{2}} .
$$

The radial node intensity function is thus given by

$$
\lambda(r)=4 \lambda \pi r-\frac{4 \lambda \pi r^{3}}{R^{2}} .
$$

Using the probability generating functional (pgfl) to calculate the Laplace transform, we obtain

$$
\begin{aligned}
\mathcal{L}_{I_{R}}(s) & =\exp \left(-\mathbb{E}_{h}\left[\int_{0}^{R}\left(1-\exp \left(-s h r^{-\alpha}\right)\right) \lambda(r) \mathrm{d} r\right]\right) \\
& \triangleq \exp \left(-\mathbb{E}_{h}[A(h)]\right) .
\end{aligned}
$$

Conditioning on the channel gain $h$,

$$
\begin{aligned}
A(h)= & \exp \left(-\int_{0}^{R}\left(1-e^{-s h r^{-\alpha}}\right) \mathrm{d}\left(2 \pi \lambda r^{2}-\frac{\lambda \pi r^{4}}{R^{2}}\right)\right) \\
= & \exp \left(-\left(1-e^{-s h R^{-\alpha}}\right) \lambda \pi R^{2}\right) \\
& \cdot \exp \left(2 \pi \lambda \alpha s h \int_{0}^{R} r^{-\alpha+1} e^{-s h r^{-\alpha}} \mathrm{d} r\right) \\
& \cdot \exp \left(-\frac{\alpha s h \lambda \pi}{R^{2}} \int_{0}^{R} r^{-\alpha+3} e^{-s h r^{-\alpha}} \mathrm{d} r\right) .
\end{aligned}
$$

Letting $R \rightarrow \infty$ and using the L'Hopital's rule, we obtain that

$$
\lim _{R \rightarrow \infty} \frac{1-e^{-s h R^{-\alpha}}}{R^{-2}}=\frac{\alpha \operatorname{sh} R^{-\alpha-1} e^{-s h R^{-\alpha}}}{-2 R^{-3}} \stackrel{(a)}{=} 0,
$$

where $(a)$ holds for $\alpha>2$, and

$$
\begin{aligned}
\lim _{R \rightarrow \infty} \frac{\alpha \operatorname{sh} \lambda \pi}{R^{2}} \int_{0}^{R} r^{-\alpha+3} e^{-s h r^{-\alpha}} \mathrm{d} r \\
=\alpha \operatorname{sh} \lambda \pi \lim _{R \rightarrow \infty} \frac{R^{-\alpha+2} e^{j \omega R^{-\alpha}}}{2}=0,
\end{aligned}
$$

Therefore, we have

$$
\lim _{R \rightarrow \infty} A(h)=\exp \left(2 \pi \lambda \alpha s h \int_{0}^{R} r^{-\alpha+1} e^{-s h r^{-\alpha}} \mathrm{d} r\right) .
$$

Plugged back into (20), Proposition 1 is then proved.

Comparing (17) with [10, (18)], we notice that in an asymptotically large area, the interference generated by RWP nodes is equivalent to the interference generated by 


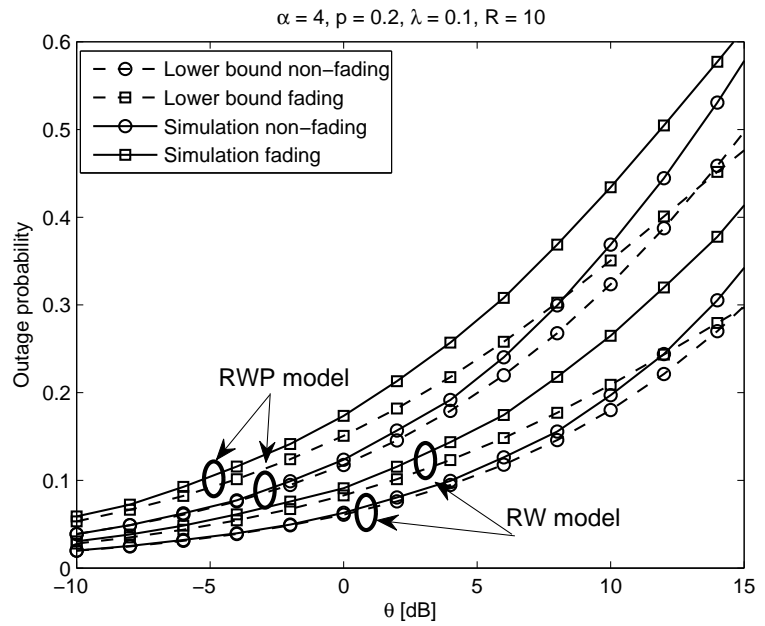

Fig. 3: Simulation results versus the corresponding lower bounds of the outage probability for different fading and mobility models.

nodes of uniformly mobility with doubled node intensity. Without fading, the outage probability $(\alpha=4)$ is given by

$$
p_{o}^{\mathrm{nf}}(\theta)=\mathbb{P}\left(I>\theta^{-1}\right)=\operatorname{erf}\left(p \pi^{\frac{3}{2}} \sqrt{\theta} \lambda\right),
$$

where $\operatorname{erf}(x)=2 \int_{0}^{x} e^{-t^{2}} \mathrm{~d} t / \sqrt{\pi}$ is the error function ${ }^{4}$.

Fig. 4 shows the outage probabilities for RWP nodes with different radii $R$ by simulations versus the asymptotic bound. The bound, which is exact for $R \rightarrow \infty$, is calculated using (21). The simulation curves approach the bound as $R$ increases. Hence, (21) can be viewed as the upper bound and the asymptotic expression of the outage probability for large $R$.

Since $\mathbb{E}\left[h^{\delta}\right]=\Gamma(1+\delta)$ for Rayleigh fading, the outage probability in Rayleigh fading environment is

$$
p_{o}^{\mathrm{f}}(\theta)=1-\mathcal{L}_{I}(\theta)=1-\exp \left(-\frac{2 p \pi^{2} \lambda \delta \theta^{\delta}}{\sin (\pi \delta)}\right) .
$$

The same extra factor 2 is obtained as we compare (22) to the homogeneous case [27, (6)], which confirms that RWP mobility increases interference.

\subsection{Tightness of the outage lower bound}

In this part, we evaluate the tightness of the outage lower bound we have obtained in finite networks. We have the following proposition.

Proposition 2. When $\theta \rightarrow 0$, the lower bound of the outage probability has the following relationship

$$
\underline{p}(\theta) \lesssim p_{o}(\theta)
$$

where "¿" means "smaller than" with asymptotic equality.

Proof: It is obvious that $p(\theta) / p_{o}(\theta)<1$. What we need to prove is $\underline{p}(\theta) \sim p_{o}(\theta)$, when $\theta \rightarrow 0$. First

4. In the expression, we have $\lambda$ for RWP and $\lambda / 2$ for uniform mobility models.

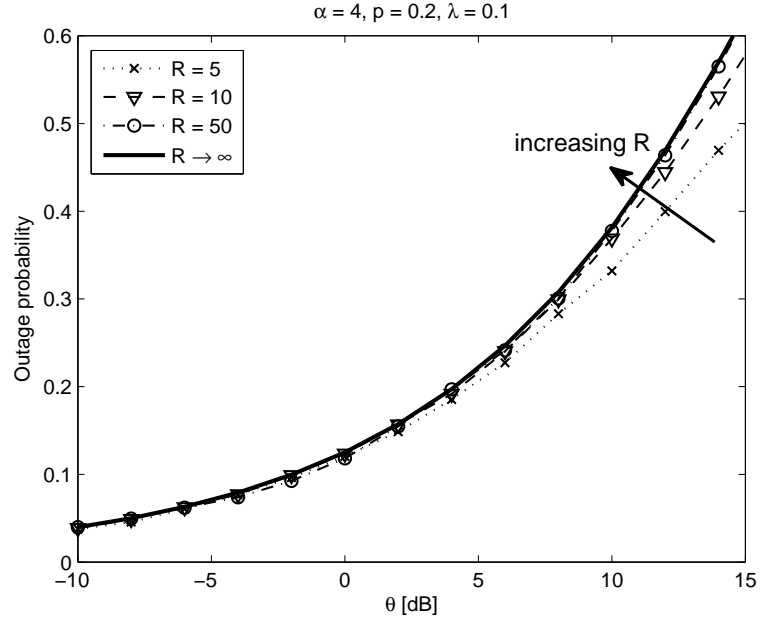

Fig. 4: The outage probabilities under the RWP mobility with different radii $R$. Channel has no multi-path fading. The bound (solid curve) is calculated analytically using (21). Other curves with finite $R$ are simulation results.

we consider the case without multi-path fading. With similar steps in [10], the ccdf of interference distribution in the infinite case is given as

$$
F_{I}(x)=\frac{1}{\pi} \sum_{k=1}^{\infty} \frac{\Gamma(\alpha k)}{k !}\left(\frac{2 \lambda p \pi \Gamma(1-\delta)}{x^{\delta}}\right)^{k} \sin (k \pi(1-\alpha)) .
$$

The term $2 \lambda$ in (24) instead of $\lambda$ in [10, (23)] is the difference between RWP and uniform mobility cases. Then

$$
\begin{aligned}
\lim _{\theta \rightarrow 0} \frac{\underline{p}^{\mathrm{nf}}(\theta)}{p_{o}^{\mathrm{nf}}(\theta)} & =\lim _{\theta \rightarrow 0} \frac{1-\exp \left(-p \lambda \pi\left(2 \theta^{\delta}-\frac{\theta^{2 \delta}}{R^{2}}\right)\right)}{\frac{1}{\pi} \sum_{k=1}^{\infty} \frac{\Gamma(\alpha k)}{k !}\left(2 \lambda p \pi \Gamma(1-\delta) \theta^{\delta}\right)^{k}} \\
& \stackrel{(a)}{=} \lim _{\theta \rightarrow 0} \frac{p \lambda \pi^{2}\left(2 \delta \theta^{\delta-1}-\frac{2 \delta}{R^{2}}\right) e^{-p \lambda \pi\left(2 \theta^{\delta}-\frac{\theta^{2 \delta}}{R^{2}}\right)}}{\sum_{k=1}^{\infty} \frac{\Gamma(\alpha k)}{k !} \delta k \theta^{\delta k-1}(2 \lambda p \pi \Gamma(1-\delta))^{k}} \\
& \stackrel{(b)}{=} 1,
\end{aligned}
$$

where $(a)$ holds because of the L'Hopital's rule; $(b)$ holds because of the dominance of the term for $k=1$ in the Taylor series expansion.

From (16) and (22), we have for the Rayleigh fading case that

$$
\lim _{\theta \rightarrow 0} \frac{p^{\mathrm{f}}(\theta)}{p_{o}^{\mathrm{f}}(\theta)} \stackrel{(a)}{=} \lim _{\theta \rightarrow 0} \frac{\int_{0}^{\infty} \frac{y}{\theta(y+1)^{2}} f_{I_{1}}(y / \theta) \mathrm{d} y}{\frac{2 p \pi^{2} \lambda \delta^{2}}{\sin (\pi \delta)} \exp \left(-\frac{2 p \pi^{2} \lambda \delta \theta^{\delta}}{\sin (\pi \delta)}\right)}=1,
$$

where $(a)$ holds because of the L'Hopital's rule.

\section{TEMPORAL CORRELATION OF INTER- FERENCE AND OUTAGE}

The interference statistics of mobile networks in a single time slot has been studied in the previous section, with concrete results for the interference and outage statistics. However, only investigating the interference in a single time slot is insufficient to design 


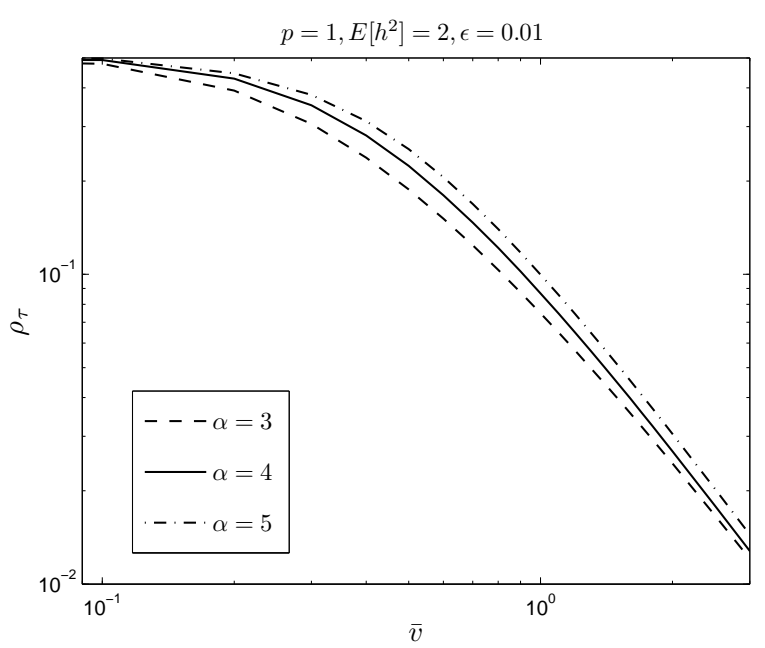

Fig. 5: The temporal correlation coefficient $\rho_{\tau}$ versus the mean speed $\bar{v}$ with different path loss exponent $\alpha$ for the CIM model.

the retransmission and routing schemes in wireless networks, since the interference is temporally and spatially correlated. Such correlation, which is caused by the locations of mobile nodes, affects retransmission and routing strategies greatly. For example in an ARQ (Automatic Repeat reQuest) retransmission mechanism, a packet is retransmitted after a timeout or after a negative acknowledgment (NACK) received. Intuitively when a link is in outage and correlation is high, blind retransmissions lead to a higher failure rate than for independent interference. Quantifying the correlation is hence necessary. In this section, we consider uniform mobility models only and focus on infinite networks. We assume that $\epsilon>0$ in (5), since for $\epsilon=0$, some integrals (such as the mean interference) are infinite.

\subsection{Temporal correlation of the interference}

In this part, we analyze the temporal correlation of the interference. The spatio-temporal correlation can be treated similarly. Because of the spatial stationarity of the point process, it is sufficient to consider the interference at the origin. The total interference in (7), $I(t)$, is identically distributed for any $t \in \mathbb{N}_{0}$. We denote the temporal correlation coefficient of the interference between time $s$ and $t$ as $\rho_{\tau} \triangleq \rho_{I(t) I(s)}$, where $\tau=|t-s|$. We have the following proposition about $\rho_{\tau}$.

Proposition 3. The temporal correlation coefficient of the interferences $I(s)$ and $I(t)$, where $s \neq t$, is given by

$$
\rho_{\tau}=\frac{p \int_{\mathbb{R}^{2}} g(x) \mathbb{E}_{w_{\tau}}\left[g\left(x+\bar{v} w_{\tau}\right)\right] \mathrm{d} x}{\mathbb{E}\left[h^{2}\right] \int_{\mathbb{R}^{2}} g^{2}(x) \mathrm{d} x},
$$

where $\bar{v} w_{\tau} \triangleq\left(\bar{v} w_{\tau, 1}, \bar{v} w_{\tau, 2}\right)$ is the location difference of a node between time $s$ and $t$.

Proof: Since $I(s)$ and $I(t)$ are identically distributed, we have

$$
\rho_{\tau} \triangleq \frac{\operatorname{Cov}(I(t), I(s))}{\operatorname{Var}[I(t)]}=\frac{\mathbb{E}[I(t) I(s)]-\mathbb{E}[I(t)]^{2}}{\mathbb{E}\left[I(t)^{2}\right]-\mathbb{E}[I(t)]^{2}} .
$$

The first and second moments of the interference follow from Campbell's theorem [8]:

$$
\mu_{I} \triangleq \mathbb{E}[I(t)]=p \lambda \int_{\mathbb{R}^{2}} g(x) \mathrm{d} x,
$$

and

$$
\begin{aligned}
\zeta_{I}^{2} & \triangleq \mathbb{E}\left[I(t)^{2}\right] \\
& =p \lambda \mathbb{E}\left[h^{2}\right] \int_{\mathbb{R}^{2}} g^{2}(x) \mathrm{d} x+p^{2} \lambda^{2}\left(\int_{\mathbb{R}^{2}} g(x) \mathrm{d} x\right)^{2} .
\end{aligned}
$$

The mean product of $I(t)$ and $I(s)$, where $t \neq s$, is given by

$$
\begin{aligned}
\mathbb{E} & {[I(t) I(s)] } \\
= & \mathbb{E}\left[\sum_{x \in \Phi(t)} T_{x}(t) h_{x}(t) g(x) \sum_{y \in \Phi(s)} T_{y}(s) h_{y}(s) g(y)\right] \\
= & \mathbb{E}\left[\sum_{x \in \Phi(s)} T_{x}(t) h_{x}(t) g\left(x+\bar{v} w_{\tau}\right) \sum_{y \in \Phi(s)} T_{y}(s) h_{y}(s) g(y)\right] \\
= & \mathbb{E}\left[\sum_{x \in \Phi(s)} T_{x}(t) T_{x}(s) h_{x}(t) h_{x}(s) g\left(x+\bar{v} w_{\tau}\right) g(x)\right]+ \\
& \mathbb{E}\left[\sum_{x, y \in \Phi(s)}^{x \neq y} T_{x}(t) T_{y}(s) h_{x}(t) h_{y}(s) g\left(x+\bar{v} w_{\tau}\right) g(y)\right]
\end{aligned}
$$

where $\bar{v} w_{\tau}$ is the location difference of a node between time $s$ and $t$. Using Campbell's theorem and the independence property of the multi-path fading gain $h_{x}$, we further write (29) as

$$
\begin{aligned}
& \mathbb{E}[I(t) I(s)] \\
& =\quad p^{2}(\mathbb{E} h)^{2} \lambda \int_{\mathbb{R}^{2}} \mathbb{E}_{w_{\tau}}\left[g\left(x+w_{\tau}\right) g(x)\right] \mathrm{d} x \\
& \quad+p^{2}(\mathbb{E} h)^{2} \lambda^{2} \int_{\mathbb{R}^{2}} \int_{\mathbb{R}^{2}} \mathbb{E}_{w_{\tau}}\left[g\left(x+w_{\tau}\right) g(y)\right] \mathrm{d} x \mathrm{~d} y \\
& \stackrel{(a)}{=} \quad p^{2} \lambda \int_{\mathbb{R}^{2}} g(x) \mathbb{E}_{w_{\tau}}\left[g\left(x+w_{\tau}\right)\right] \mathrm{d} x \\
& \quad+p^{2} \lambda^{2}\left(\int_{\mathbb{R}^{2}} g(x) \mathrm{d} x\right)^{2}
\end{aligned}
$$

where $(a)$ follows from the following relationship:

$$
\begin{aligned}
p \lambda \int_{\mathbb{R}^{2}} g(x) \mathrm{d} x & =\mathbb{E}[I(0)]=\mathbb{E}[I(t)] \\
& =\mathbb{E}\left[\sum_{x \in \Phi(0)} T_{x}(t) h_{x}(t) g\left(x+\bar{v} w_{t}\right)\right] \\
& =p \lambda \int_{\mathbb{R}^{2}} \mathbb{E}_{w_{t}}\left[g\left(x+\bar{v} w_{t}\right)\right] \mathrm{d} x
\end{aligned}
$$


Therefore, Proposition 3 is proved.

Corollary 4. In d-dimensional networks, the correlation coefficient is given by

$$
\rho_{\tau}=\frac{p \int_{\mathbb{R}^{d}} g(x) \mathbb{E}_{w_{\tau}}\left[g\left(x+\bar{v} w_{\tau}\right)\right] \mathrm{d} x}{\mathbb{E}\left[h^{2}\right] \int_{\mathbb{R}^{d}} g^{2}(x) \mathrm{d} x} .
$$

Proof: Follows directly from the proof of Proposition 3.

The spatio-temporal correlation coefficient of the interference at two given locations is provided in [29, (11)]. For mobile networks, the random position difference of the nodes in different time slots needs to be averaged out. The difference between the static and mobile networks is that in a static network, the path loss $g(x)$ does not change in one realization, while $g(x(t))$ is time variant in a mobile network. The correlation coefficient is independent of the intensity $\lambda$, since the interference scales linearly with $\lambda$. We now have the following corollary about $\rho_{\tau}$.

Corollary 5. The temporal correlation coefficient $\rho_{\tau}$ is bounded by

$$
\rho_{\tau} \leqslant \frac{p}{\mathbb{E}\left[h^{2}\right]} .
$$

Denote the pdf of $w_{\tau}$ as $f_{w_{\tau}}(x, y)$. If $f_{w_{\tau}}(0,0)$ is bounded, we have

$$
\rho_{\tau} \sim C_{1} \bar{v}^{-2}, \quad \bar{v} \rightarrow \infty,
$$

where

$$
C_{1}=\frac{p f_{w_{\tau}}(0,0)\left(\int_{\mathbb{R}^{2}} g(x) \mathrm{d} x\right)^{2}}{\mathbb{E}\left[h^{2}\right] \int_{\mathbb{R}^{2}} g^{2}(x) \mathrm{d} x} .
$$

If $\forall(x, y) \neq(0,0), f_{w_{\tau}}(0,0) \geqslant f_{w_{\tau}}(x, y), \rho_{\tau}$ is bounded by

$$
\rho_{\tau} \lesssim C_{1} \bar{v}^{-2}, \quad \bar{v} \rightarrow \infty
$$

where "ふ" means "smaller than" with asymptotic equality.

Proof: Exploring $\mathbb{E}_{w_{\tau}}\left[g\left(x+\bar{v} w_{\tau}\right)\right]$ in (25) for the twodimensional case, we observe that

$$
\begin{aligned}
& \mathbb{E}_{w_{\tau}}\left[g\left(x+\bar{v} w_{\tau}\right)\right] \\
& \quad=\int_{-\infty}^{\infty} \int_{-\infty}^{\infty} \frac{f_{w_{\tau}}\left(z_{1}, z_{2}\right)}{\epsilon+\left(\left(x_{1}+\bar{v} z_{1}\right)^{2}+\left(x_{2}+\bar{v} z_{2}\right)^{2}\right)^{\frac{\alpha}{2}}} \mathrm{~d} z_{1} \mathrm{~d} z_{2}
\end{aligned}
$$

and, in turn, $\rho_{\tau}$ decreases monotonically with $\bar{v}$. Hence

$$
\rho_{\tau} \leqslant \lim _{\bar{v} \rightarrow 0} \frac{p \int_{\mathbb{R}^{2}} g(x) \mathbb{E}_{w_{\tau}}\left[g\left(x+\bar{v} w_{\tau}\right)\right] \mathrm{d} x}{\mathbb{E}\left[h^{2}\right] \int_{\mathbb{R}^{2}} g^{2}(x) \mathrm{d} x}=\frac{p}{\mathbb{E}\left[h^{2}\right]} .
$$

Rewriting (35) as

$$
\begin{aligned}
& \mathbb{E}_{w_{\tau}}\left[g\left(x+\bar{v} w_{\tau}\right)\right] \\
& =\frac{1}{\bar{v}^{2}} \int_{-\infty}^{\infty} \int_{-\infty}^{\infty} \frac{f_{w_{\tau}}\left(\frac{t_{1}}{\bar{v}}, \frac{t_{2}}{\bar{v}}\right)}{\epsilon+\left(\left(x_{1}+t_{1}\right)^{2}+\left(x_{2}+t_{2}\right)^{2}\right)^{\frac{\alpha}{2}}} \mathrm{~d} t_{1} \mathrm{~d} t_{2},
\end{aligned}
$$

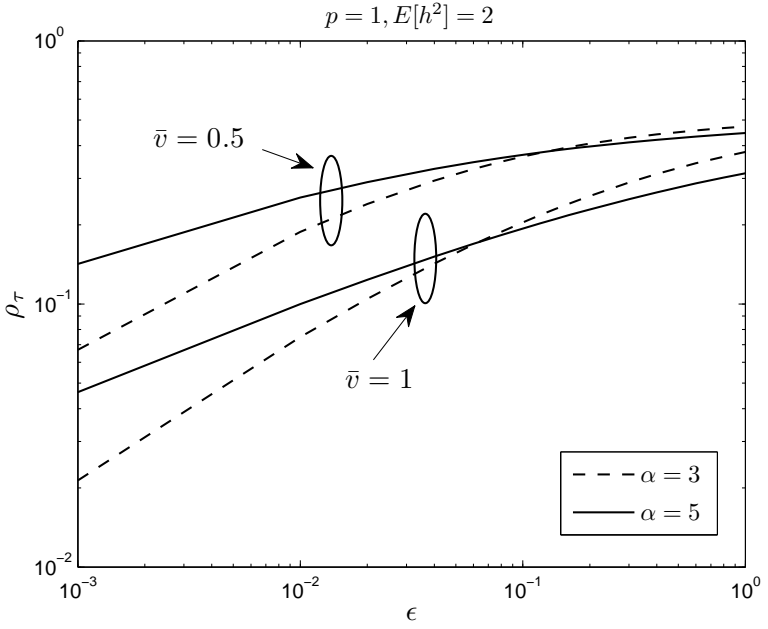

Fig. 6: The temporal correlation coefficient $\rho_{\tau}$ versus $\epsilon$ for the CIM model.

We obtain

$$
\lim _{\bar{v} \rightarrow \infty} \rho_{\tau} \bar{v}^{2}=\frac{p f_{w_{\tau}}(0,0)\left(\int_{\mathbb{R}^{2}} g(x) \mathrm{d} x\right)^{2}}{\mathbb{E}\left[h^{2}\right] \int_{\mathbb{R}^{2}} g^{2}(x) \mathrm{d} x} \triangleq C_{1}<\infty .
$$

If $\forall(x, y) \neq(0,0), f_{w_{\tau}}(0,0) \geqslant f_{w_{\tau}}(x, y), \mathbb{E}_{w_{\tau}}\left[g\left(x+\bar{v} w_{\tau}\right)\right]$ in (36) is upper bounded by

$$
\mathbb{E}_{w_{\tau}}\left[g\left(x+\bar{v} w_{\tau}\right)\right] \leqslant \frac{f_{w_{\tau}}(0,0) \int_{\mathbb{R}^{2}} g(x) \mathrm{d} x}{\bar{v}^{2}} .
$$

(32) is consistent with [29, Corollary 2] for static networks when $\bar{v} \rightarrow 0$. $f_{w_{\tau}}(0,0)<\infty$ indicates that the probability where a node returns to its original position after time interval $\tau$ is zero. Corollary 5 is valid for the uniform mobility models considered in Section 2.2.

Interestingly, the decay of $\rho_{\tau}(\bar{v})$ is always asymptotically proportional to $\bar{v}^{-2}$. Fig. 5 shows $\rho_{\tau}$ versus the mean speed $\bar{v}$ with different $\alpha$ under the CIM model. In one-dimensional networks, $\rho_{\tau}$ is asymptotically proportional to $\bar{v}^{-1}$ as we have shown in [40]. Moreover, it can be extended to a $d$-dimensional model, where $\rho_{\tau}$ is asymptotically proportional to $\bar{v}^{-d}$. The dimension $d$ equals to the degrees of freedom.

Fig. 6 shows $\rho_{\tau}$ versus $\epsilon$. When $\epsilon$ is small, $\rho_{\tau}$ increases with $\alpha$. For $\alpha$ not too close to 2, interferers close to the origin dominate the interference. Such dominance is more prominent with larger $\alpha$ and hence causes higher temporal correlation of the interference. However, $\rho_{\tau}$ decreases with $\alpha$ when $\epsilon$ is large. More nodes contribute to the interference in this case. The larger the path loss exponent, the more correlated the interference is. Moreover, the correlation coefficient $\rho_{\tau} \rightarrow 0$ as $\epsilon \rightarrow 0$. As stated in the previous section, the interference is dominated by the nearest transmitter in singular pathloss model. The interferers in $B(0, \varrho)$, where $0<\varrho \ll 1$, dominate the interference. As the nodes move, we view independent sets of interferers from $B(0, \varrho)$ in different time instants. The correlation coefficient hence goes to 
zero in the singular path-loss model. It needs to be pointed out that we only use the first and second moments of interference statistics when we capture the correlation in terms of the correlation coefficient. In singular path-loss model, even the first moment of the interference $I$ is infinity. A more powerful metric for dependence, such as mutual information, may reveal that there is indeed still a dependence as $\epsilon \rightarrow 0$.

The integral $\int_{\mathbb{R}^{2}} g(x) \mathbb{E}_{w_{\tau}}\left[g\left(x+\bar{v} w_{\tau}\right)\right] \mathrm{d} x$ in (25) depends on the mobility models. In the next several subsections, we discuss different mobility models individually.

\subsubsection{Constrained i.i.d. mobility (CIM)}

Under the CIM model, the first term in (29) can be rewritten as

$$
\begin{gathered}
\mathbb{E}\left[\sum_{x \in \Phi(s)} T_{x}(t) T_{x}(s) h_{x}(t) h_{x}(s) g\left(x+\bar{v} w_{\tau}\right) g(x)\right] \\
\stackrel{(a)}{=} p^{2} \mathbb{E}\left[\sum_{x \in \Psi} g\left(x+\bar{v} w_{t}\right) g\left(x+\bar{v} w_{s}\right)\right] \\
\stackrel{(b)}{=} p^{2} \lambda \int_{\mathbb{R}^{2}} \mathbb{E}_{w_{s}}\left[g\left(x+\bar{v} w_{s}\right)\right]^{2} \mathrm{~d} x,
\end{gathered}
$$

where $(a)$ follows from the independence of $T_{x}(t)$ and $T_{x}(s)$; (b) follows from the fact that $w_{t}, w_{s}$ are i.i.d. and uniformly at random in $B\left(x, R_{\mathrm{CIM}}\right)$. Thus, $\rho_{\tau, \mathrm{CIM}}$ is given by

$$
\rho_{\tau, \mathrm{CIM}}=\frac{p \int_{\mathbb{R}^{2}} \mathbb{E}_{w_{s}}\left[g\left(x+\bar{v} w_{s}\right)\right]^{2} \mathrm{~d} x}{\mathbb{E}\left[h^{2}\right] \int_{\mathbb{R}} g^{2}(x) \mathrm{d} x} .
$$

Using the limit in (34) as an upper bound, we have the following corollary about $\rho_{\tau, \mathrm{CIM}}$.

Corollary 6. The temporal correlation coefficient under the CIM model $\rho_{\tau, \text { CIM }}$ is upper bounded by

$$
\rho_{\tau, \mathrm{CIM}} \lesssim \frac{p}{\mathbb{E}\left[h^{2}\right]} \cdot \min \left\{1, \frac{\delta \pi \epsilon^{\delta}}{(1-\delta) R_{\mathrm{CIM}}^{2} \sin (\pi \delta) \bar{v}^{2}}\right\},
$$

where $R_{\mathrm{CIM}}=45 \pi / 128$.

Proof: Since we have

$$
\begin{gathered}
f_{w_{\tau}}(0,0)=\frac{1}{\pi R_{\mathrm{CIM}}^{2}}, \\
\int_{\mathbb{R}^{2}} g(x) \mathrm{d} x=\frac{2 \pi^{2}}{\alpha \epsilon^{1-2 / \alpha} \sin (2 \pi / \alpha)},
\end{gathered}
$$

and

$$
\int_{\mathbb{R}^{2}} g^{2}(x) \mathrm{d} x=\frac{2(\alpha-2) \pi^{2}}{\alpha^{2} \epsilon^{2-2 / \alpha} \sin (2 \pi / \alpha)},
$$

(38) follows from Corollary 5 after several steps of calculation.

Fig. 7 shows the numerical evaluation of $\rho_{\tau, \text { CIM }}$ from (37) (solid curves) together with the upper bound from (38) (dashed curves). The numerical evaluation converge to the bound fast as $\bar{v}$ increases and thus provides a tight upper bound for all $\alpha>2$.

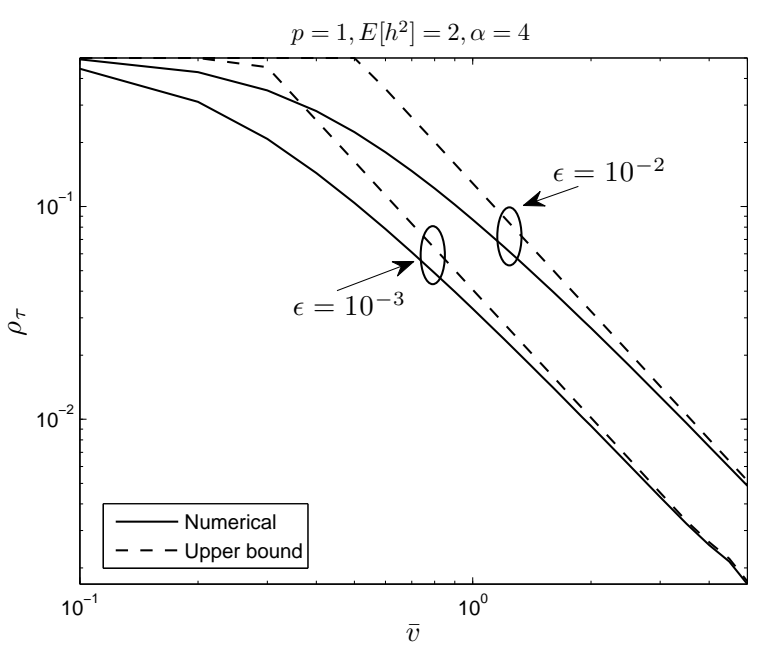

Fig. 7: Numerical evaluation (from (37)) of the temporal correlation coefficient $\rho_{\tau}$ versus average node speed $\bar{v}$ with the corresponding upper bound (from (38)). The mobility model is CIM.

From (37) and (38), we find that the temporal correlation remains stationary over time under the CIM model, since $\rho_{\tau, \text { CIM }}$ is independent of $\tau$. This observation matches with the property of the CIM model. For the Nakagami- $m$ fading model, we have $\mathbb{E}\left[h^{2}\right]=\frac{m+1}{m}$. In particular, $\mathbb{E}\left[h^{2}\right]=2$ for Rayleigh fading $(m=1)$, and $\mathbb{E}\left[h^{2}\right]=1$ for no fading $(m \rightarrow \infty) . \rho_{\tau, \mathrm{CIM}}$ increases with $m$, as well as with the MAC scheme parameter $p$. Conversely, both fading and random MAC scheduling schemes reduce the temporal correlation of interference.

\subsubsection{Random walk (RW)}

Under the RW model, we focus on the temporal correlation of the interference between two successive time slots, i.e., $\rho_{1}$. By a similar derivation as for the CIM model, we have the following corollary about $\rho_{1, \mathrm{RW}}$.

Corollary 7. The temporal correlation coefficient under the $R W$ model $\rho_{1, \mathrm{RW}}$ is upper bounded by

$$
\rho_{1, \mathrm{RW}} \lesssim \frac{p}{\mathbb{E}\left[h^{2}\right]} \cdot \min \left\{1, \frac{4 \delta \pi \epsilon^{\delta}}{9(1-\delta) \sin (\pi \delta) \bar{v}^{2}}\right\} .
$$

Proof: Since we have

$$
f_{w_{1}}(0,0)=\frac{1}{\pi R_{\mathrm{RW}}^{2}},
$$

the calculation is straightforward following the proof of Corollary 6.

Fig. 8 displays the numerical evaluation of $\rho_{1, \mathrm{RW}}$ from (25) and its upper bound from (39). The upper bound converges fast to the numerical curves.

\subsubsection{Discrete-time Brownian motion (BM)}

Under the BM model, we have

$$
w_{\tau}=\sum_{i=1}^{\tau} w(i) \stackrel{(d)}{=} \sqrt{\tau} w_{0},
$$




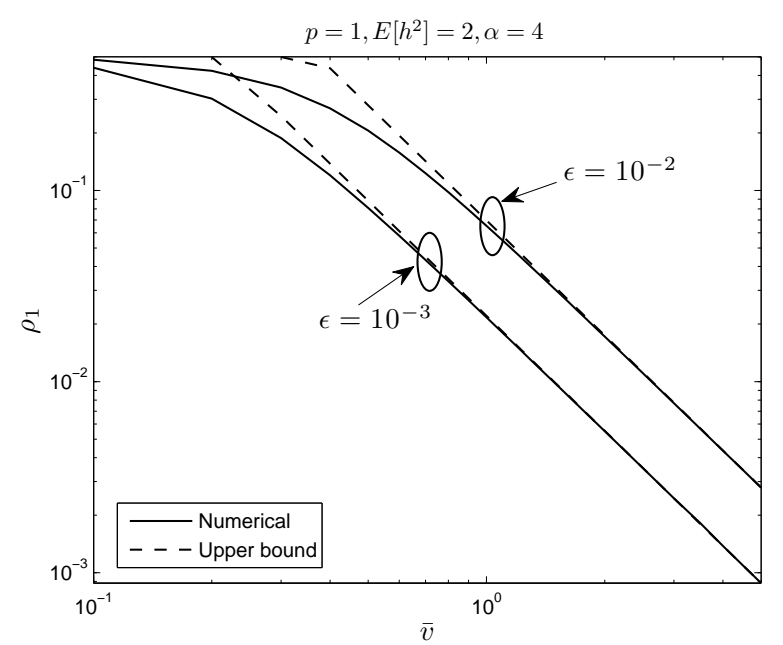

Fig. 8: Numerical evaluation (from (25)) of the temporal correlation coefficient $\rho_{1}$ versus average node speed $\bar{v}$ with the corresponding upper bound (from (39)). The mobility model is RW.

where $\stackrel{(d)}{=}$ denotes equality in distribution and $w_{0}$ is a two dimensional Gaussian random variable, i.e., $\mathcal{N}\left(0, \sigma^{2} \mathbf{I}\right)$, where $\mathbf{I}$ is a 2-by-2 identity matrix. Hence, (25) can be rewritten as

$$
\rho_{\tau, \mathrm{BM}}=\frac{p \int_{\mathbb{R}^{2}} g(x) \mathbb{E}_{w_{0}}\left[g\left(x+\sqrt{\tau} \bar{v} w_{0}\right)\right] \mathrm{d} x}{\mathbb{E}\left[h^{2}\right] \int_{\mathbb{R}} g^{2}(x) \mathrm{d} x} .
$$

Fig. 9 plots $\rho_{1}$ versus the mean speed of nodes $\bar{v}$ under three mobility models. As we observe from the figure, $\rho_{1, \mathrm{CIM}}$ and $\rho_{1, \mathrm{BM}}$ are approximately equal. $\rho_{1, \mathrm{RW}}$ decays slightly faster with the increase of $\bar{v}$. The difference between them is less than 0.02 . Therefore the mean speed $\bar{v}$ rather than the specific nature of the mobility models dominates the temporal correlation of the interference. For $\tau>1$, we have the following corollary about $\rho_{\tau, \mathrm{BM}}$.

Corollary 8. When the time difference $\tau \rightarrow \infty$, the temporal correlation coefficient under the BM model is

$$
\rho_{\tau, \mathrm{BM}} \sim C_{2} \tau^{-1}
$$

where

$$
C_{2}=\frac{C_{1}}{\bar{v}^{2}}=\frac{\pi^{2} \epsilon^{2 / \alpha}}{2(\alpha-2) \sin (2 \pi / \alpha) \bar{v}^{2}},
$$

and $\rho_{\tau, \mathrm{BM}}$ is upper bounded by

$$
\rho_{\tau, \mathrm{BM}} \lesssim \frac{p}{\mathbb{E}\left[h^{2}\right]} \cdot \min \left\{1, \frac{\pi^{2} \epsilon^{2 / \alpha}}{2(\alpha-2) \sin (2 \pi / \alpha) \tau \bar{v}^{2}}\right\} .
$$

Proof: Based on Corollary 5, (41) and (42) follow from (40) after a few elementary steps.

Similarly, we can show that $\rho_{\tau, \mathrm{BM}}$ is asymptotically proportional to $\tau^{-d / 2}$ in $d$-dimensional networks.

\subsection{Outage correlation}

In the design of retransmission schemes in wireless networks, it is often assumed that outage events are independent across time for the sake of mathematical simplicity. However, due to the temporal correlation of interference, link outage events are temporally correlated as well. Intuitively speaking, a link in outage at a given time indicates a higher outage probability in the next several time slots because of the correlation of network geometry. Such correlation affects retransmission and routing schemes greatly and thus need to be quantified. The correlation of link outage in static networks is examined in [29]. In this section, we discuss the temporal correlation of outage in mobile networks. Let $A_{t}$ denotes the event that the link is in outage at time $t$, i.e., $A_{t} \triangleq$ $\left\{\operatorname{SIR}(t)=\frac{h(t)}{I(t)}<\theta\right\}$, where the distance of the desired link is normalized to one as indicated in Section 2.1. The joint probability of events $A_{s}$ and $A_{t}$ is given in (43), where $(a)$ follows from the independence of $h(s)$ and $h(t)$; (b) follows from the identical distribution of $I(t)$ and $I(s) ;(c)$ follows from the averaging of $T_{x}$ and $h_{x}$; $(d)$ holds from the pgfl of the PPP.

The direct evaluation of (43) seems hopeless, since the joint distribution of the two correlated random variables $I(t)$ and $I(s)$ is hard to obtain. However, an approximation can be obtained by exploring the functional relationship between $I(s)$ and $I(t)$. Regression analysis is a statistical technique for investigating and modeling relationships between two correlated variables [41], [42]. In that way, $I(t)$ is expressed as a linear combination of $I(s)$, a component orthogonal to $I(s)$, a constant component, and a random error component. We introduce a random variable $\tilde{I}(t)$ such that $\tilde{I}(t)$ and $I(s)$ are identically distributed and independent, i.e., $\mathbb{E}[I(s) \tilde{I}(t)]=0$. The covariance of $\tilde{I}(t)$ and $I(t)$ is $\operatorname{Cov}(\tilde{I}(t), I(s))=K\left(\zeta_{I}-\mu_{I}^{2}\right)$, where $K$ is an unknown parameter. Since $I(s)$ and $I(t)$ are correlated, the multiple linear regression model that describes this relationship is given by

$$
I(t)=\beta_{0}+\beta_{1} I(s)+\beta_{2} \tilde{I}(t)+\xi,
$$

where $\beta_{0}, \beta_{1}$, and $\beta_{2}$ are regression coefficients to be determined, and the residual $\xi$ is a random error.

If $I(t)$ in (43) is replaced by the expression in (44), the joint distribution of $I(t)$ and $I(s)$ is avoided. The method of minimum mean square error (MMSE) estimation is used to estimate the unknown parameters $\left\{\beta_{i}, i=0,1,2\right\}$ in (44). Geometrically, the linear model (44) describes a two-dimensional estimation space of two orthogonal regressors $I(s)$ and $\tilde{I}(t)$ and provides a linear representation $\hat{I}(t)$ that is closest to $I(t)$. The error term $\xi$ in the model has the property of zero mean, i.e., $\underset{\tilde{I}}{\mathbb{E}}[\xi]=0$, and is mutually independent with $I(t)$ and $\tilde{I}(t)$, i.e., $\mathbb{E}[I(s) \xi]=\mathbb{E}[\tilde{I}(t) \xi]=0$. We denote the covariance matrix of $I(s)$ and $\tilde{I}(t)$ as $\mathbf{S}=\left(\zeta_{I}-\mu_{I}^{2}\right)$ I. From [41], we have the coefficients that minimize the mean square error as

$$
\left[\begin{array}{l}
\beta_{1} \\
\beta_{2}
\end{array}\right]=\mathbf{S}^{-1}\left[\begin{array}{c}
\operatorname{Cov}(I(t), I(s)) \\
\operatorname{Cov}(I(t), \tilde{I}(t))
\end{array}\right]=\left[\begin{array}{c}
\rho_{\tau} \\
K
\end{array}\right],
$$




$$
\begin{aligned}
\mathbb{P}\left(A_{s}, A_{t}\right)= & \mathbb{P}(h(s)<\theta I(s), h(t)<\theta I(t)) \\
\stackrel{(a)}{=} & \mathbb{E}_{I(s), I(t)}[(1-\exp (-\theta I(s)))(1-\exp (-\theta I(t)))], \\
\stackrel{(b)}{=} & 1-2 \mathbb{E}[\exp (-\theta I(t))]+ \\
& \mathbb{E}\left[\exp \left(-\theta \sum_{x \in \Phi(s)}\left(T_{x}(s) h_{x}(s) g(x)+T_{x}(t) h_{x}(t) g\left(x+\bar{v} w_{\tau}\right)\right)\right)\right] \\
\stackrel{(c)}{=} & 1-2 \mathcal{L}_{I}(\theta)+ \\
& \mathbb{E}\left[\prod_{x \in \Phi(s)}\left(\frac{p}{1+\theta g(x)}+1-p\right)\left(\frac{p}{1+\theta g\left(x+\bar{v} w_{\tau}\right)}+1-p\right)\right] \\
\stackrel{(d)}{=} & 1-2 \mathcal{L}_{I}(\theta)+\mathbb{E}_{w_{\tau}}\left[\operatorname { e x p } \left(-\lambda \int_{\mathbb{R}^{2}} 1-\left(\frac{p}{1+\theta g(x)}+1-p\right) .\right.\right. \\
& \left.\left.\left(\frac{p}{1+\theta g\left(x+\bar{v} w_{\tau}\right)}+1-p\right) \mathrm{d} x\right)\right] .
\end{aligned}
$$

and

$$
\beta_{0}=\mu_{I}\left(1-\rho_{\tau}-K\right),
$$

respectively, where $\rho_{\tau}$ is the correlation coefficient we have obtained in Section 4.1.

Taking the expectation of (44) on both sides, we obtain that $K \in\left[0,1-\rho_{\tau}\right]$. Here, the mobility models themselves are not crucial. $\rho_{\tau}$ is sufficient to approximate the joint outage probability $\mathbb{P}\left(A_{s}, A_{t}\right)$ in (43), which is given by

$$
\begin{aligned}
\mathbb{P}\left(A_{s}, A_{t}\right) & \\
= & \mathbb{E}[(1-\exp (-\theta I(s))) \\
& \left.\cdot\left(1-\exp \left(-\theta\left(\beta_{0}+\rho_{\tau} I(s)+K \tilde{I}(t)+\xi\right)\right)\right)\right] \\
= & 1-\mathbb{E}[\exp (-\theta I(s))] \\
& -\mathbb{E}\left[\exp \left(-\theta \rho_{\tau} I(s)\right)-\exp \left(-\theta\left(1+\rho_{\tau}\right) I(s)\right)\right] \\
& \cdot \mathbb{E}\left[\exp \left(-\theta\left(\beta_{0}+K \tilde{I}(t)\right)\right)\right] \mathbb{E}[\exp (-\theta \xi)] \\
(a) & 1-\mathcal{L}_{I}(\theta)-\left(\mathcal{L}_{I}\left(\rho_{\tau} \theta\right)-\mathcal{L}_{I}\left(\left(1+\rho_{\tau}\right) \theta\right)\right) . \\
& \mathcal{L}_{I}(\theta K) \cdot \exp \left(-\theta\left(1-\rho_{\tau}-K\right) \mu_{I}\right),
\end{aligned}
$$

where $(a)$ follows from the fact that $\mathbb{E}[\exp (-\theta \xi)] \geqslant 1$ by Jensen's inequality. The upper bound of $\mathbb{P}\left(A_{s}, A_{t}\right)$ in (47) is a function of $K$. With different $K,(47)$ provides a set of upper bounds of the joint outage probability $\mathbb{P}\left(A_{s}, A_{t}\right)$. Next, we find the tightest upper bound by searching the $K^{*}$ such that the upper bound of (47) is minimized. We then have the following proposition about $\mathbb{P}\left(A_{s}, A_{t}\right)$.

Proposition 9. The joint outage probability $\mathbb{P}\left(A_{s}, A_{t}\right)$ is upper bounded by

$$
\begin{aligned}
\mathbb{P}\left(A_{s}, A_{t}\right) \leqslant & 1-\mathcal{L}_{I}(\theta)-\mathcal{L}_{I}\left(\left(1-\rho_{\tau}\right) \theta\right) \\
& \cdot\left(\mathcal{L}_{I}\left(\rho_{\tau} \theta\right)-\mathcal{L}_{I}\left(\left(1+\rho_{\tau}\right) \theta\right)\right) .
\end{aligned}
$$

Proof: Under the non-singular path-loss model, the Laplace transform of the interference $\mathcal{L}_{I}(s)$ for 2dimensional Poisson networks is re-calculated as

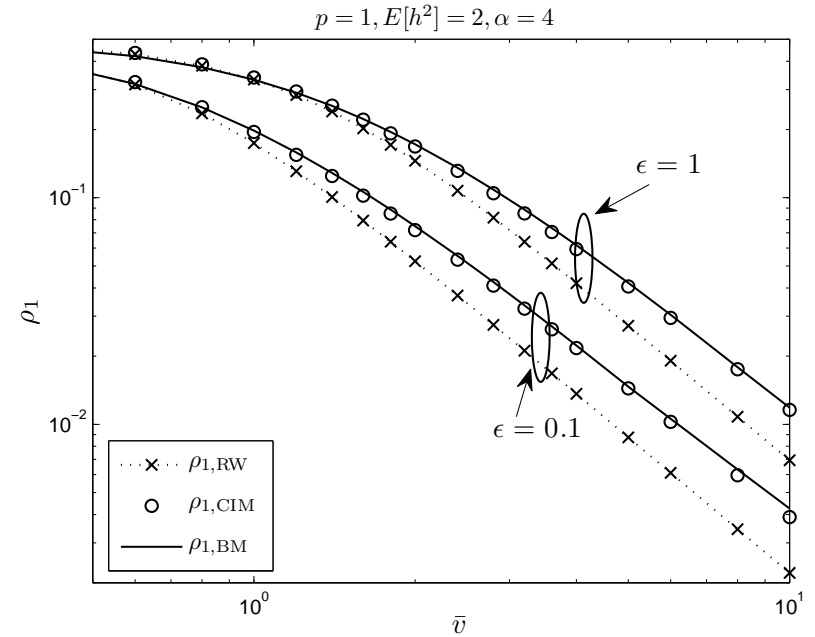

Fig. 9: The interference correlation coefficient $\rho_{1}$ versus the mean speed $\bar{v}$ under three mobility models.

$$
\begin{aligned}
& \mathcal{L}_{I}(s) \\
& =\mathbb{E}_{\Phi, h_{x}, T_{x}}\left[\exp \left(-s \sum_{x \in \Phi} T_{x} h_{x} g(x)\right)\right] \\
& =\mathbb{E}_{\Phi}\left[\prod_{x \in \Phi} \mathbb{E}_{h_{x}, T_{x}}\left[\exp \left(-s T_{x} h_{x} g(x)\right)\right]\right] \\
& =\exp \left(-\pi p \lambda \mathbb{E}_{h}\left(\int_{0}^{\infty}\left(1-\exp \left(-\frac{s h}{\epsilon+x^{1 / \delta}}\right)\right) \mathrm{d} x\right)\right) \\
& =\exp \left(-p \lambda \delta \pi^{2} \csc (\pi \delta) \frac{s}{(s+\epsilon)^{1-\delta}}\right),
\end{aligned}
$$

and

$$
\mu_{I}=\frac{\delta \pi^{2} p \lambda}{\epsilon^{1-\delta} \sin (\pi \delta)}
$$

Finding the $K^{*}$ that minimizes the upper bound of (47) 
is equivalent to finding the $K^{*}$ such that

$$
K^{*}=\underset{K \in\left[0,1-\rho_{\tau}\right]}{\arg \sup }\left\{f(K) \triangleq \frac{K}{\epsilon^{1-2 / \alpha}}-\frac{2 K}{(\theta K+\epsilon)^{1-2 / \alpha}}\right\} .
$$

It is easy to see that $f(K)$ is monotonically increasing over $K \in\left[0,1-\rho_{\tau}\right]$. Hence,

$$
K^{*}=\beta_{2}=1-\rho_{\tau} .
$$

Proposition 9 is then proved.

Corollary 10. The conditional outage probability $\mathbb{P}\left(A_{t} \mid A_{s}\right)$ is upper bounded by

$$
\mathbb{P}\left(A_{t} \mid A_{s}\right) \leqslant 1-\frac{\mathcal{L}_{I}\left(\left(1-\rho_{\tau}\right) \theta\right)\left(\mathcal{L}_{I}\left(\rho_{\tau} \theta\right)-\mathcal{L}_{I}\left(\left(1+\rho_{\tau}\right) \theta\right)\right)}{1-\mathcal{L}_{I}(\theta)} .
$$

Proof: Since

$$
\mathbb{P}\left(A_{t} \mid A_{s}\right)=\frac{\mathbb{P}\left(A_{s}, A_{t}\right)}{\mathbb{P}\left(A_{t}\right)},
$$

the proof is straightforward from Proposition 9.

Corollary 11. The conditional outage probability $\mathbb{P}\left(A_{t} \mid \bar{A}_{s}\right)$ is upper bounded by

$\mathbb{P}\left(A_{t} \mid \bar{A}_{s}\right)=\frac{\mathbb{P}\left(\bar{A}_{s}, A_{t}\right)}{\mathbb{P}\left(\bar{A}_{s}\right)} \leqslant 1-\frac{\mathcal{L}_{I}\left(\left(1+\rho_{\tau}\right) \theta\right) \mathcal{L}_{I}\left(\left(1-\rho_{\tau}\right) \theta\right)}{\mathcal{L}_{I}(\theta)}$.

Proof: Similar to the proof of Proposition 9,

$$
\begin{aligned}
\mathbb{P}\left(\bar{A}_{s}, A_{t}\right) & =\mathbb{P}(h(s)>\theta I(s), h(t)<\theta I(t)) \\
& =\mathbb{E}_{I(s), I(t)}[\exp (-\theta I(s))(1-\exp (-\theta I(t)))] \\
& \leqslant \mathcal{L}_{I}(\theta)-\mathcal{L}_{I}\left(\left(1+\rho_{\tau}\right) \theta\right) \mathcal{L}_{I}\left(\left(1-\rho_{\tau}\right) \theta\right) .
\end{aligned}
$$

The corollary is then proved.

For comparison purposes, $\mathbb{P}\left(A_{t} \mid A_{s}\right)$ in static networks in [29] is re-calculated under the non-singular path-loss model as

$$
\mathbb{P}\left(A_{t} \mid A_{s}\right)=1-\mathcal{L}_{I}(\theta)+\frac{(B-1) \mathcal{L}_{I}^{2}(\theta)}{1-\mathcal{L}_{I}(\theta)},
$$

and

$$
\mathbb{P}\left(A_{t} \mid \bar{A}_{s}\right)=1-B \mathcal{L}_{I}(\theta),
$$

where

$$
\begin{aligned}
B & =\exp \left(\lambda p^{2} \int_{\mathbb{R}^{2}}\left(\frac{\theta g(x)}{1+\theta g(x)}\right)^{2} \mathrm{~d} x\right) \\
& =\exp \left(\frac{2 \pi^{2}(\alpha-2) \theta^{2} \lambda p^{2}}{\alpha^{2}(\epsilon+\theta)^{2-2 / \alpha} \sin (2 \pi / \alpha)}\right) .
\end{aligned}
$$

Fig. 10 and 11 display the simulation evaluations of the conditional outage probability versus the threshold $\theta$ and the MAC scheme parameter $p$, respectively, together with the tight upper bounds from (53) and (54). The CIM model is used in the simulation. The unconditional outage probability $\mathbb{P}\left(A_{t}\right)$ is always less than $\mathbb{P}\left(A_{t} \mid A_{s}\right)$. The outage evaluation in a single time slot ignores the information about previous link states

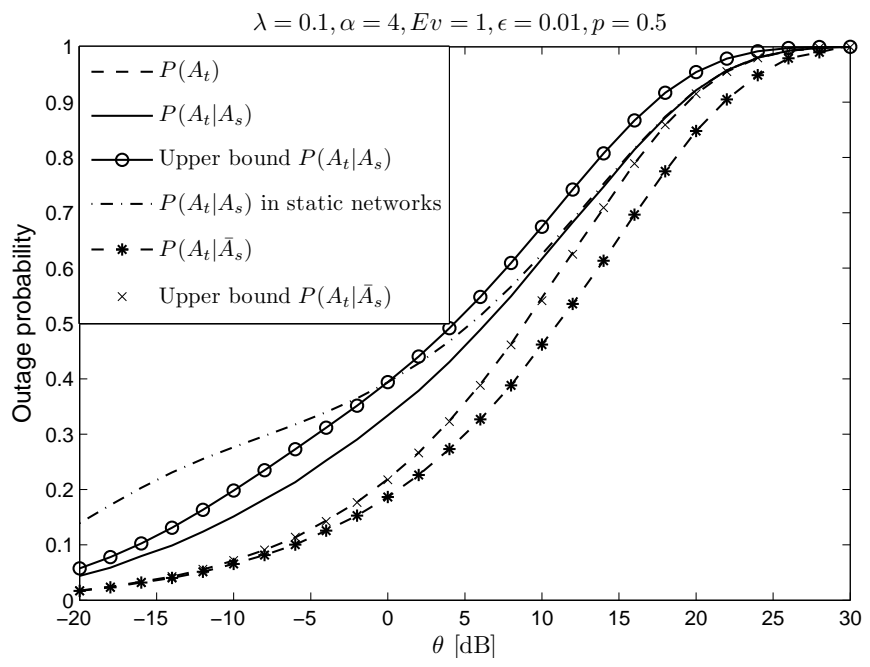

Fig. 10: Conditional outage probability $\mathbb{P}\left(A_{t} \mid A_{s}\right)$ together with unconditional outage probability $\mathbb{P}\left(A_{t}\right)$ versus the threshold $\theta$. The circles are the upper bound of $\mathbb{P}\left(A_{t} \mid A_{s}\right)$ from (53); the solid-line curve is the exact expression via simulations; the crosses are the upper bound of $\mathbb{P}\left(A_{t} \mid \bar{A}_{s}\right)$ from (54).

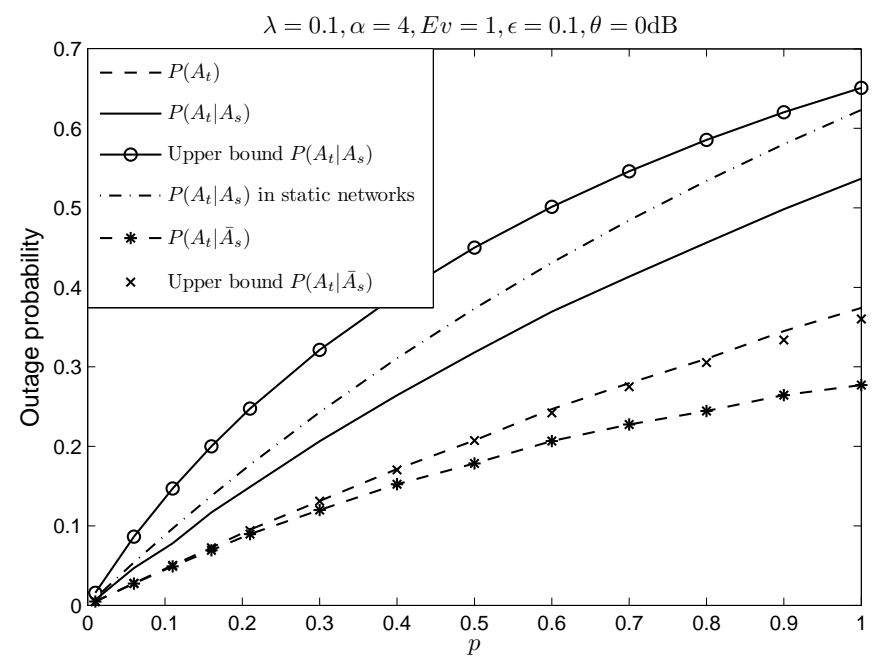

Fig. 11: Conditional outage probability $\mathbb{P}\left(A_{t} \mid A_{s}\right)$ together with unconditional outage probability $\mathbb{P}\left(A_{t}\right)$ versus the MAC scheme parameter $p$.

and thus provides an over-optimistic evaluation of the network performance. Conversely, $\mathbb{P}\left(A_{t}\right)>\mathbb{P}\left(A_{t} \mid \bar{A}_{s}\right)$ as expected. When the threshold increases, the difference between $\mathbb{P}\left(A_{t} \mid A_{s}\right), \mathbb{P}\left(A_{t} \mid \bar{A}_{s}\right)$, and $\mathbb{P}\left(A_{t}\right)$ shrinks, since the link is more likely to be in outage and thus the conditioning does not make a difference.

\section{Conclusions}

In this paper, we have treated macroscopic mobility from a large-scale fading perspective. Fluctuations of the path loss induced by mobility constitute another type of fading in wireless channels besides multi-path effects. To make the difference clear, we may speak 
of fading induced by microscopic mobility (multi-path fading) and fading induced by macroscopic mobility. Using this insight, we have characterized the interference distributions in mobile networks. The nearest-interferer approximation provides a tight lower bound on the outage probability. The RWP model leads to increased interference.

Furthermore, we have quantified the temporal correlation of the interference and outage in mobile networks in terms of the correlation coefficient $\rho$ and the conditional outage probability, respectively. We have shown that in $d$-dimensional networks, $\rho$ decreases asymptotically inversely proportionally with the $d$-th power of the average node speed. Multi-path fading and random MAC schemes also reduce the interference correlation. The interference correlation coefficient is a key ingredient when we explore the outage correlation in wireless networks by quantifying the conditional outage probability, where a linear regression model is used for bounding. Given that the link is in outage in the present time instant, we have shown that it has a larger probability of being in outage in the next several time instants. The retransmission strategy needs to be smart (correlation-aware) when the link is in outage, since blind retransmission leads to an even higher failure rate.

\section{ACKNOWLEDGMENTS}

The partial support of NSF (grants CCF 728763, CNS 1016742) and the DARPA/IPTO IT-MANET program (grant W911NF-07-1-0028) is gratefully acknowledged.

\section{References}

[1] D. Tse and P. Viswanath, Fundamentals of wireless communication. Cambridge University Press, 2005.

[2] M. Haenggi, J. G. Andrews, F. Baccelli, O. Dousse, and M. Franceschetti, "Stochastic Geometry and Random Graphs for the Analysis and Design of Wireless Networks," IEEE Journal on Selected Areas in Communications, vol. 27, pp. 1029-1046, Sept. 2009.

[3] F. Baccelli, Stochastic Geometry and Wireless Networks. Volume IIapplications. Foundations and Trends in Networking (NOW Publishers), vol. 4, no. 1-2, pp. 1-312, 2009.

[4] M. Chiang, P. Hande, T. Lan, and C. W. Tan, Power control in wireless cellular networks. Foundations and Trends in Networking (NOW Publishers), vol. 2, no. 4, pp. 381-533 2009.

[5] M. Haenggi and R. K. Ganti, Interference in Large Wireless Networks. Foundations and Trends in Networking (NOW Publishers), vol. 3, no. 2, pp. 127-248, 2009.

[6] R. M. Metcalfe and D. R. Boggs, "Ethernet: Distributed packet switching for local computer networks," Communications of the ACM, vol. 19, no. 7, pp. 395-404, 1976.

[7] L. Kleinrock and F. Tobagi, "Packet switching in radio channels: Part I-carrier sense multiple-access modes and their throughputdelay characteristics," IEEE Transactions on Communications, vol. 23, no. 12, pp. 1400-1416, 1975.

[8] D. Stoyan, W. S. Kendall, and J. Mecke, Stochastic geometry and its applications. Wiley Chichester, 1995.

[9] M. Penrose, Random geometric graphs. Oxford University Press, USA, 2003.

[10] E. Sousa and J. Silvester, "Optimum transmission ranges in a direct sequence spread-spectrum multihop packet radio network," IEEE Journal on Selected Areas in Communications, vol. 8, pp. 762-771, Jun 1990.

[11] E. Sousa, "Interference modeling in a direct-sequence spread-spectrum packet radio network," IEEE Transactions on Communications, vol. 38, no. 9, pp. 1475-1482, 1992.
[12] F. Baccelli, B. Blaszczyszyn, and P. Muhlethaler, "An Aloha protocol for multihop mobile wireless networks," IEEE Transactions on Information Theory, vol. 52, no. 2, pp. 421-436, 2006.

[13] M. Zorzi and S. Pupolin, "Optimum transmission ranges in multihop packet radio networks in the presence of fading," IEEE Transactions on Communications, vol. 43, no. 7, pp. 2201-2205, 2002.

[14] S. Srinivasa and M. Haenggi, "Distance Distributions in Finite Uniformly Random Networks: Theory and Applications," IEEE Transactions on Vehicular Technology, vol. 59, pp. 940-949, Feb. 2010.

[15] R. K. Ganti and M. Haenggi, "Interference and Outage in Clustered Wireless Ad Hoc Networks," IEEE Transactions on Information Theory, vol. 55, pp. 4067-4086, Sept. 2009.

[16] R. K. Ganti and M. Haenggi, "Interference in Ad Hoc Networks with General Motion-Invariant Node Distributions," in 2008 IEEE International Symposium on Information Theory (ISIT'08), (Toronto, Canada), July 2008.

[17] R. Giacomelli, R. K. Ganti, and M. Haenggi, "Outage Probability of General Ad Hoc Networks in the High-Reliability Regime," IEEE/ACM Transactions on Networking, To appear.

[18] R. K. Ganti, J. G. Andrews, and M. Haenggi, "High-SIR Transmission Capacity of Wireless Networks with General Fading and Node Distribution," IEEE Transactions on Information Theory, vol. 57, pp. 3100-3116, May 2011.

[19] C. Lee and M. Haenggi, "Interference and Outage in Doubly Poisson Cognitive Networks," in International Conference on Computer Communications and Networks (ICCCN), 2010.

[20] H. Nguyen, F. Baccelli, and D. Kofman, "A stochastic geometry analysis of dense IEEE 802.11 networks," in IEEE INFOCOM, pp. 1199-1207, 2007.

[21] O. Dousse, M. Franceschetti, and P. Thiran, "On the throughput scaling of wireless relay networks," IEEE/ACM Transactions on Networking (TON), vol. 14, no. 6, pp. 2756-2761, 2006.

[22] R. K. Ganti and M. Haenggi, "Spatial Analysis of Opportunistic Downlink Relaying in a Two-Hop Cellular System," IEEE Transactions on Communications, accepted.

[23] F. Baccelli and C. Bordenave, "The radial spanning tree of a Poisson point process," Annals of Applied Probability, vol. 17, no. 1, pp. 305-359, 2007.

[24] M. Haenggi, "On Routing in Random Rayleigh Fading Networks," IEEE Transactions on Wireless Communications, vol. 4, pp. 1553-1562, July 2005.

[25] F. Baccelli, B. Blaszczyszyn, and P. Muhlethaler, "On the performance of time-space opportunistic routing in multihop mobile ad hoc networks," in 7th International Symposium on Modeling and Optimization in Mobile, Ad Hoc, and Wireless Networks (WiOpt'09), Berlin, 2008.

[26] S. P. Weber, X. Yang, J. G. Andrews, and G. D. Veciana, "Transmission capacity of wireless ad hoc networks with outage constraints," IEEE Transactions on Information Theory, vol. 51, no. 12, pp. 4091-4102, 2005.

[27] M. Haenggi, "Outage, Local Throughput, and Capacity of Random Wireless Networks," IEEE Transactions on Wireless Communications, vol. 8, pp. 4350-4359, Aug 2009.

[28] R. K. Ganti and M. Haenggi, "Limit of the Transport Capacity of a Dense Wireless Network," Journal of Applied Probability, vol. 47, pp. 886-892, Sept. 2010.

[29] R. K. Ganti and M. Haenggi, "Spatial and temporal correlation of the interference in ALOHA ad hoc networks," IEEE Communications Letters, vol. 13, no. 9, pp. 631-633, 2009.

[30] R. K. Ganti and J. G. Andrews, "Correlation of link outage in low-mobility spatial wireless networks," in Asilomar Conference on Signals, Systems, and Computers, (Pacific Grove), Nov. 2010.

[31] Z. Kong and E. M. Yeh, "On the latency for information dissemination in mobile wireless networks," in Proceedings of the 9th ACM International Symposium on Mobile Ad Hoc Networking and Computing (MobiHoc), Hong Kong SAR, China, May 2008.

[32] T. Camp, J. Boleng, and V. Davies, "A survey of mobility models for ad hoc network research Research," Wireless Communications and Mobile Computing, vol. 2, no. 5, pp. 483-502, 2002.

[33] S. Bandyopadhyay, E. J. Coyle, and T. Falck, "Stochastic properties of mobility models in mobile ad hoc networks," IEEE Transactions on Mobile Computing, vol. 6, no. 11, pp. 1218-1229, 2007.

[34] C. Bettstetter, "Mobility modeling in wireless networks: categorization, smooth movement, and border effects," ACM SIGMOBILE Mobile Computing and Communications Review, vol. 5, no. 3, p. 66, 2001. 
[35] C. Bettstetter, G. Resta, and P. Santi, "The node distribution of the random waypoint mobility model for wireless ad hoc networks," IEEE Transactions on Mobile Computing, vol. 2, no. 3, pp. 257-269, 2003.

[36] C. Bettstetter, "On the connectivity of ad hoc networks," The Computer Journal, vol. 47, no. 4, p. 432, 2004

[37] E. Hyytia, P. Lassila, and J. Virtamo, "Spatial node distribution of the random waypoint mobility model with applications," IEEE Transactions on Mobile Computing, vol. 5, no. 6, pp. 680-694, 2006.

[38] M. Haenggi, "A Geometric Interpretation of Fading in Wireless Networks: Theory and Applications," IEEE Transactions on Information Theory, vol. 54, pp. 5500-5510, Dec 2008.

[39] P. Nain, D. Towsley, B. Liu, Z. Liu, and F. Inria, "Properties of random direction models," in IEEE INFOCOM, vol. 3, 2005.

[40] Z. Gong and M. Haenggi, "Temporal Correlation of the Interference in Mobile Random Networks," in IEEE International Conference on Communications (ICC'11), (Kyoto, Japan), June 2011.

[41] D. C. Montgomery, E. A. Peck, and G. G. Vining, Introduction to linear regression analysis. New York: Wiley-Interscience, 2001.

[42] S. Chatterjee and A. S. Hadi, Regression analysis by example. John Wiley and Sons, 2006. 